\title{
Alkaline phosphatase and neonatal hyperbilirubinemia - correlations and cutoff values
}

\author{
Vlad Dima ${ }^{1}$, Andreea Calomfirescu-Avramescu' ${ }^{1}$, Luminita Paduraru², Simona Vladareanu ${ }^{3}$ \\ ${ }^{1}$ Filantropia Clinical Hospital, Bucharest, Romania \\ 2"Gr. T. Popa" University of Medicine and Pharmacy, lasi, Romania \\ 3"Carol Davila" University of Medicine and Pharmacy, Bucharest, Romania
}

\begin{abstract}
Introduction. Since the second half of the eighteenth century, neonatal jaundice has been a widely debated topic and the first records on neonatal jaundice were made in antiquity. Although it is a common pathology, in some cases it can be severe, leading to bilirubin encephalopathy. In recent years, many studies have tried to correlate and to identify predictive factors for the occurrence of severe hyperbilirubinemia. The most recent associations have been made with the level of alkaline phosphatase because it is a ubiquitous enzyme with an important role in hepatic metabolism.

Material and method. A study on a group of 250 full-term, normal-weight newborns without associated pathologies was performed. The aim of the study was to determine the alkaline phosphatase values at birth, transcutaneous bilirubin values at 24 and 48 hours after delivery and the need of phototherapy as treatment.

Results and conclusions. A high statistical correlation was demonstrated between elevated alkaline phosphatase values, measured in the umbilical cord blood and neonatal jaundice. A cutoff value for alkaline phosphatase correlated with the need for phototherapy was also determined.
\end{abstract}

Keywords: neonatal jaundice, alkaline phosphatase, hyperbilirubinemia

\section{INTRODUCTION}

Jaundice is a common problem in neonatal period, sometimes overlooked, which can cause severe neurological sequelae in the absence of appropriate treatment. About $85 \%$ of full-term newborns and most premature infants develop clinical jaundice (1). Jaundice represents the yellowing of the skin and mucous membranes due to the accumulation of bile pigments (bilirubin) in the blood and deposition in the tissues. Specifically, jaundice occurs when the liver cannot remove enough bilirubin from the blood (2). Total serum bilirubin results from the difference between bilirubin production and excretion. The clinical manifestations of jaundice range from discrete yellow coloration of the skin and sclera to the most severe forms of acute or chronic bilirubin encephalopathy (3). In recent years, many attempts have been made to establish clinical correlations between bilirubin levels and other enzymes in order to determine early predictable factors for severe hyperbilirubinemia (4). Thus, altered alkaline phosphatase (AP) values have been a major research topic in recent decades, studied in relation with many disorders. Alkaline phosphatase plays an important role in the hepatic metabolism and bone development (5). It is used both as a biomarker that helps diagnose several pathologies, such as hepatitis or osteomalacia, and recently as a substitution therapy in some diseases such as perinatal hypophosphatemia and ulcerative-necrotic enterocolitis in preterm infants (6).

\section{MATERIAL AND METHOD}

This was a prospective cohort study conducted at Filantropia Hospital between January and June 2019. The study included 250 full-term normal neonates, delivered after 37 weeks of gestation, without other 
associated pathologies. $\mathrm{ABO}$ or $\mathrm{Rh}$ system incompatibilities, hemolytic and metabolic diseases were excluded. A sample of approximately $2 \mathrm{ml}$ of venous cord blood was collected from each infant in order to perform a full blood count and to determine the blood group, $\mathrm{Rh}$ type and alkaline phosphatase level.

A database was created and analyzed using IBM SPSS 20. The examined variables were nominal (gender, type of birth, need for phototherapy) and continuous or discrete quantitative variables (gestational age, Apgar score at 1 minute and 5 minutes, birth weight, alkaline phosphatase, transcutaneous bilirubin values at 24 and 48 hours and number of days of hospitalization). For quantitative variables we determined the central tendency (the mean and median) and the variability (standard deviation), while for qualitative variables, absolute frequencies and percentages/proportions were used.

To test the statistical significance, we used the $t$ test for variables with parametrical distribution and Mann-Whitney test for non-parametrical variables. The significance level was considered for $\mathrm{p}$ value $<0.05$.

To determine the cutoff value (the value with the highest sensitivity and the lowest value for 1-Specificity), ROC (receiver operator chart) curves were derived which show the totality of the potential combinations of specificity and sensitivity for each value of the parameter (in this case alkaline phosphatase). The accuracy indicator of the test used, respectively the area under the curve (AUC) was 0.98. The closer this indicator is to 1 , the better the test distinguishes between those who will need phototherapy and those who will not need phototherapy. The $95 \% \mathrm{CI}$ for AUC was 0.97 and 0.99 .

The study was conducted with the approval of the Fliantropia Clinical Hospital Ethical Committee and the informed consent was obtained from the legal relatives.

\section{RESULTS}

The study included 250 healthy newborns who were monitored for alkaline phosphatase levels, determined from umbilical cord venous blood immediately after birth and subsequent evolution of transcutaneous bilirubin levels. Alkaline phosphatase values ranged from $145 \mathrm{U} / 1$ to $602 \mathrm{U} / 1$ with a mean of $282.69 \mathrm{U} / 1$ (SD +/- $62.15 \mathrm{U} / 1)$ and a median of $298 \mathrm{U} / 1$. The step of the histogram was $25 \mathrm{U} / 1$ with the maximum number of subjects being in the range of 325-350 U/1.

The newborns were divided into two groups according to the need for phototherapy. For $87.60 \%$ of the subjects $(n=219)$ there was no need for treatment, while for the remaining $12.40 \%(\mathrm{n}=31)$ phototherapy was required.

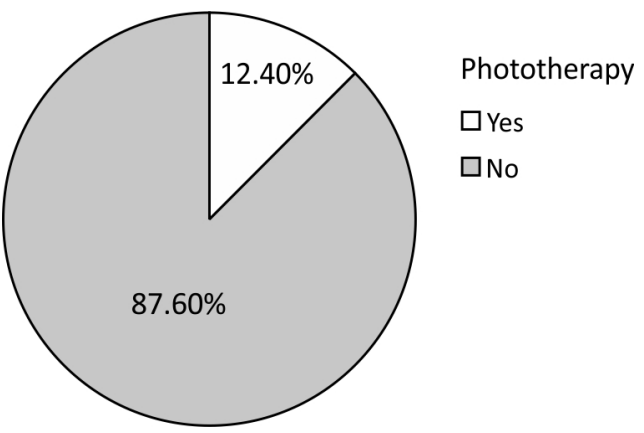

FIGURE 1. The percentage of neonates with and without phototherapy

For neonates who did not require phototherapy, gestational age at delivery ranged from 37 to 41 weeks with a mean of 38.9 weeks (SD +/-1.36 weeks) and a median of 39 weeks. In neonates receiving phototherapy, gestational age at delivery ranged from 37 to 41 weeks with a mean of 39.1 weeks (SD +/- 1.33 weeks) and a median of 39 weeks. Box-plot graph and Kolmogorov-Smirnov normality tests show that the values have a parametric distribution. The $t$ test for independent samples showed that there was no statistically significant difference between the mean values of gestational age of the groups with and without therapy $(\mathrm{t}=-1.04, \mathrm{p}=0.29)$.

In neonates without phototherapy, birth weight ranged between 2,500 and $4,050 \mathrm{~g}$ with a mean of $3,043.1 \mathrm{~g}(\mathrm{SD}+/-303.23 \mathrm{~g})$ and a median of $3,050 \mathrm{~g}$. In newborns for whom phototherapy was required, birth weight ranged between 2,550 and $3,950 \mathrm{~g}$ with a mean of $3101.6 \mathrm{~g}$ (SD +/- $298.74 \mathrm{~g}$ ) and a median of $3,150 \mathrm{~g}$. The $\mathrm{t}$ test, applied for independent samples, showed that there was no statistically significant difference between birth weight values depending on the presence/absence of treatment $(\mathrm{t}=-1.01, \mathrm{p}=0.31)$.

In neonates who did not require phototherapy, alkaline phosphatase values ranged from $145 \mathrm{U} / 1$ to 417 $\mathrm{U} / 1$, with a mean of $268.9 \mathrm{U} / 1$ (SD +/- $48.61 \mathrm{U} / 1)$ and a median of $288.7 \mathrm{U} / 1$. In neonates requiring phototherapy, alkaline phosphatase values were between $320 \mathrm{U} / 1$ and $602 \mathrm{U} / 1$ with a mean of $379.7 \mathrm{U} / 1$ (SD +/- $61.52 \mathrm{U} / 1$ ) and a median of $356 \mathrm{U} / 1$ (Figure 2). We applied the $t$ test which showed that the average alkaline phosphatase values in neonates who required phototherapy were significantly higher compared to those in neonates for whom no phototherapy was performed $(t=-11.47, p=.00$ with a significant size of Cohen's effect of 1.99).

Transcutaneous bilirubin (TB) values at 24 hours ranged between 4.1 and $7.8 \mathrm{mg} / \mathrm{dl}$ with a mean of 5.8 $\mathrm{mg} / \mathrm{dl}(\mathrm{SD}+/-0.79 \mathrm{mg} / \mathrm{dl})$ and a median of $5.8 \mathrm{mg} / \mathrm{dl}$. 


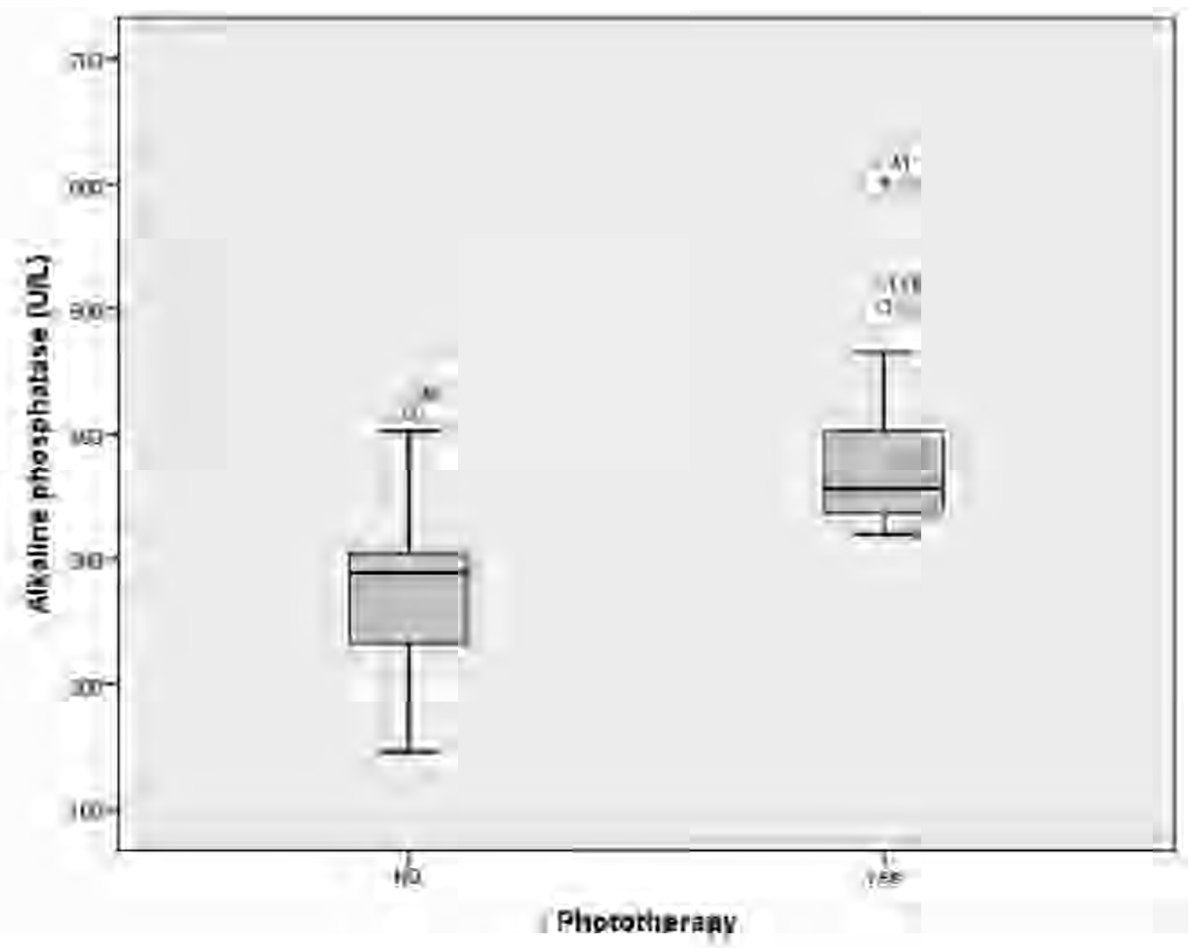

FIGURE 2. Box-plot chart for alkaline phosphatase values in the groups with and without phototherapy

TB values at 48 hours were between 5.3 and $14 \mathrm{mg} / \mathrm{dl}$ with a mean of $10.51 \mathrm{mg} / \mathrm{dl}(\mathrm{SD}+/-1.54 \mathrm{mg} / \mathrm{dl})$ and a median of $10.30 \mathrm{mg} / \mathrm{dl}$. Outlier values were observed among the TB values at $48 \mathrm{~h}$ (Figure 3 ). The $\mathrm{t}$ test for independent samples showed that the mean TB values at 48 hours was statistically significantly higher than that measured at 24 hours $(\mathrm{T}=-43.04, \mathrm{p}=.00)$.
Based on the results obtained with a high statistical significance, the cutoff value of alkaline phosphatase was determined. Thus, for the area under the curve of 0.98 , the cutoff value for alkaline phosphatase was $319.55 \mathrm{U} / 1$ (sensitivity $97 \%$ and 1 -specificity $=.06$ (false positive) so specificity $=94 \%$ ).

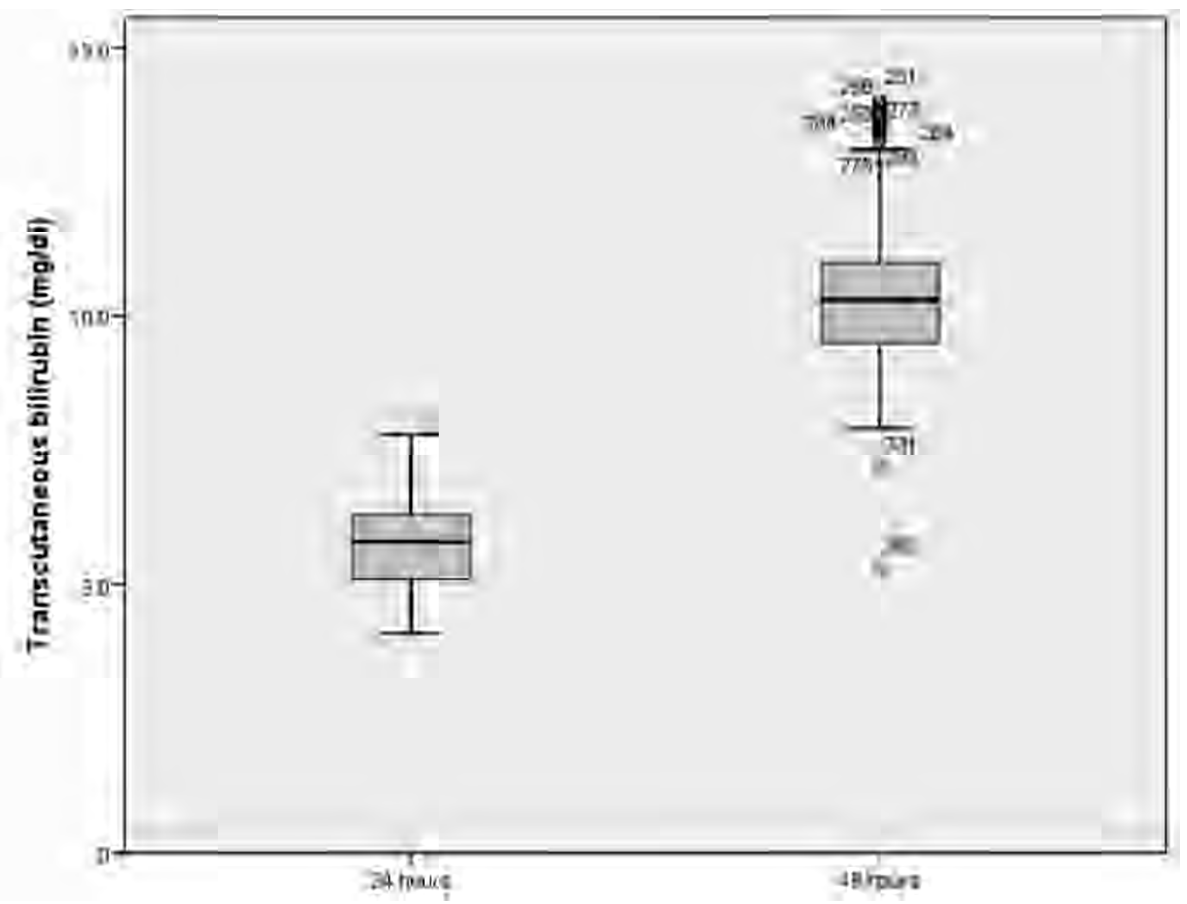

FIGURE 3. Transcutaneous bilirubin values at 24 and 48 hours 


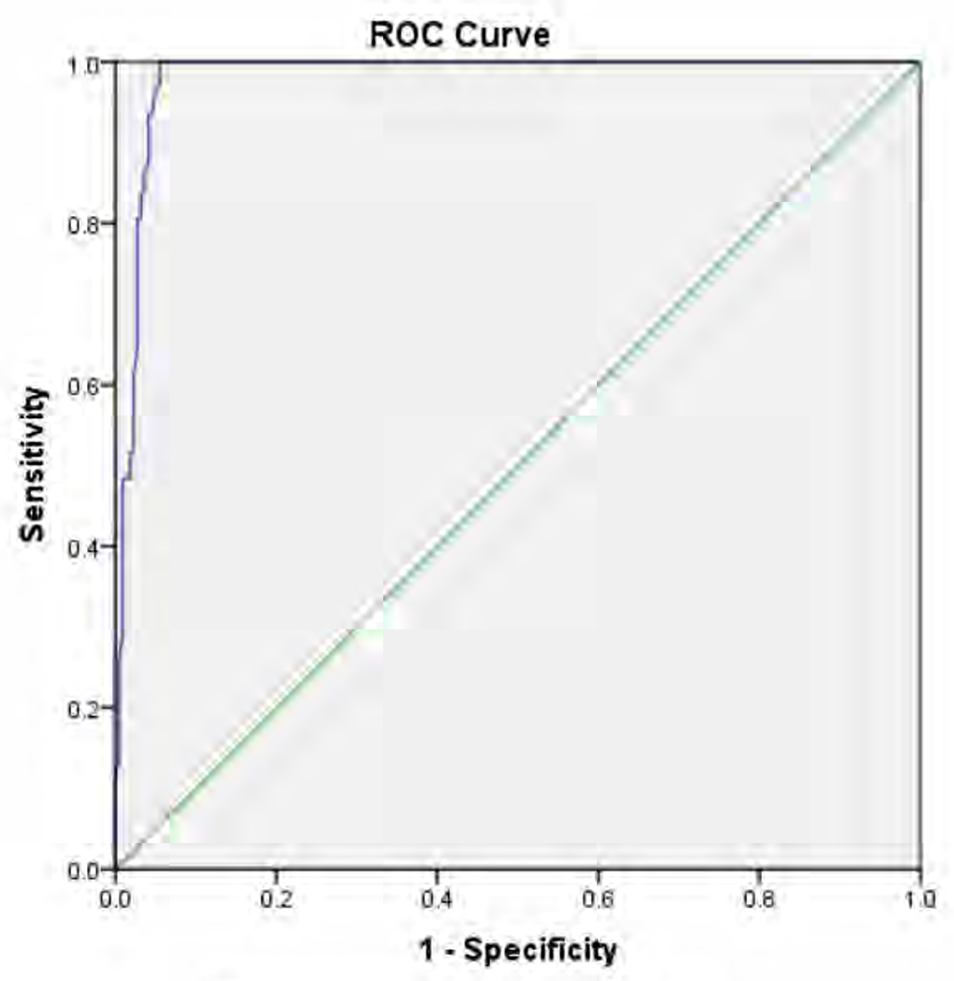

Diagonal segments are produced by ties.

FIGURE 4. ROC curve of alkaline phosphatase

\section{DISCUSSIONS}

Jaundice is a common disorder in neonates, usually without major implications, but which can rarely lead to serious complications. Elevated bilirubin levels require immediate treatment to prevent its potential toxic effects. In recent years, studies have focused on the non-invasive measurement of bilirubin, respectively the measurement of bilirubin value in the skin (transcutaneous bilirubin). It has been shown that here is a good correlation between transcutaneous and serum bilirubin values in mixed populations and infants irrespective of gestational age, including preterm neonates at less than 32 weeks gestation $(7,8)$ and this method significantly reduces the need of venous punctures (9). Therefore, in our study we correlated the values of transcutaneous bilirubin measured at 24 and 48 hours of life, with the values of alkaline phosphatase.

The values of transcutaneous bilirubin indicating the need for phototherapy treatment were the same as those in the study conducted by Fouzas et al. in 2010 (10).

The alkaline phosphatase level is greatly influenced by growth, changes in bone metabolism and in the hepatobiliary system and it depends on age and sex, especially during childhood and puberty. Due to this variability, it is important to determine when phosphatase values are pathologically or physiologically increased, correlated to age and sex. In 2017, Zierk and collaborators conducted a study for the correct interpretation of alkaline phosphatase values according to age and sex (11). The alkaline phosphatase reference values used in our study were adapted to the age and sex of the newborn.

Pathologically increased values are encountered in: biliary obstruction, disorders involving increased osteoblast activity (such as bone Paget's disease), elevated calcium levels (such as hyperparathyroidism), vitamin D3 deficiency, hepatocyte damage, celiac disease. Placental phosphatase isoenzyme may increase in malignant tumors such as seminomas (12). Lower phosphatase levels are less common.

In recent years, several studies have been conducted in an attempt to determine a correlation between the neonatal jaundice and alkaline phosphatase values. In 2017, Al Assal and colleagues published the first study on the correlation of alkaline phosphatase values in the umbilical cord blood and the evolution of neonatal jaundice (13). The study included 200 full-term newborns and the results showed that all infants with elevated alkaline phosphatase levels required treatment for hyperbilirubinemia.

In our study, a statistically significant difference was observed between the alkaline phosphatase values collected from the umbilical cord between the two 
groups. In the group without phototherapy the values were between $145 \mathrm{IU} / 1$ and 417 IU/1 while in the group that needed treatment the values were more increased between $320 \mathrm{IU} / 1$ and $602 \mathrm{IU} / 1$. The mean values in the treatment group were $379.73+/-61.52 \mathrm{IU} / 1$, which although high is different from the values of the study of Eid et al (14) in which the value was 352.11 $\pm 53.49 \mathrm{IU} / 1$ or of Abbasian et al. (15), in which the value was $314.34 \pm 122.42 \mathrm{IU} / 1$. These values support the hypothesis of Nalbantoglu et al. (16) that correlate elevated alkaline phosphatase values with neonatal hyperbilirubinemia and the need for phototherapy. The difference between his study and our study is that they measured alkaline phosphatase values at 6 hours of life of the newborn, and our values were recorded at birth.

Like the presented studies, our research focused on determining a cutoff value of alkaline phosphatase to use as early as possible as a predictive factor for the subsequent evolution of jaundice.

Also in 2019, Elmonem and colleagues conducted a study on 100 neonates born after 35 weeks of gestation showing that alkaline phosphatase levels can be a good predictor of severe jaundice and can predict its onset (17). Also in our study we found a statistically significant difference in the number of days of hospi-

\section{REFERENCES}

1. Babaei H, Alipour AA, Hemmati M, Ghaderi M, Rezaei M. Effect of white plastic cover around the phototherapy unit on hyperbilirubinemia in full term neonates. Iran J Pediatrics. 2013;23:143-8.

2. Hay W, Levin M, Deterding R, Abzug M. Current diagnosis \& treatment pediatrics 24th edition. Lange Medical Book, 2018.

3. Erlinger S, Arias IM, Dhumeaux D. Inherited disorders of bilirubin transport and conjugation: new insights into molecular mechanisms and consequences. Gastroenterology 2014;146(7):1625-38.

4. American Academy of Pediatrics Subcommittee hyperbilirubinaemia. Management of hyperbilirubinaemia in the newborn infant 35 or more week of gestation. Pediatrics 2004;114(1):297-316.

5. Done SL. Fetal and neonatal bone health: update on bone growth and manifestations in health and disease. Pediatr Radiol. 2012; 42(Suppl 1):S158-76.

6. Kampanatkosol R, Thomson T, Habeeb O, et al. The relationship between reticulated platelets, intestinal alkaline phosphatase, and necrotizing enterocolitis. J Pediatr Surg. 2014;49:273.

7. DeLuca D, Engle W, Jackson G. Transcutaneous bilirubinometry: Hepatology research and clinical developments. New York: Nova Biomedical, 2013.

8. Nagar G, Vandermeer B, Campbell S, et al. Reliability of transcutaneous bilirubin devices in preterm infants: A systematic review. Pediatrics 2013;132:871.

9. Maisels MJ. Noninvasive measurements of bilirubin. Pediatrics 2012; 129(4):779.

10. Fouzas S, Mantagou L, Skylogianni E, Mantagos S, Varvarigou A. Transcutaneous Bilirubin Levels for the First 120 Postnatal Hours in Healthy Neonates. Pediatrics 2010;125(1):e52-e57. talization, which was higher in the group of newborns who needed phototherapy. This conclusion is the same as in the study by Satrya et al. (18), in which he described a longer hospitalization period of about 2-3 days in the treatment group.

This current research has the advantage of including a significant number of patients (250 newborns) because it was conducted in a grade 3 maternity hospital. The limitations of the study were the inclusion of only normal weight patients at term and the short duration of research.

\section{CONCLUSIONS}

The current study shows that high values of alkaline phosphatase are associated with neonatal hyperbilirubinemia and consequently with the need for phototherapy. Beyond the early identification of newborns at risk of developing severe forms of neonatal jaundice requiring phototherapy, the results of the present study might have an impact on shortening the average length of hospital stay for healthy newborns at term. Although the calculated cutoff value can be an important and applicable predictable marker, it requires further research on a larger population and over a longer period of time.

Conflict of interest: none declared Financial support: none declared

11. Zierk J, Arzideh F, Haeckel R, Cario H, Frühwald MC et al. Pediatric reference intervals for alkaline phosphatase. Clin Chem Lab Med. 2017;55(1):102-110.

12. Buchet R, Millan JL, Magne D. Multisystemic functions of alkaline phosphatases. Methods Mol Biol. 2013;1053:27-51.

13. Al Assal H, Hablas H, Afia A, Khedr M, El kzaz M. Utility of Cord Blood Alkaline Phosphatase Enzyme as a Predictor of Significant Neonatal Jaundice in Well Term Infants. N Y Sci J. 2017;10(4):70-74.

14. Eid SR, Mohamed AG, Okda HT, Abdel Salam HM. Evaluation of cord blood alkaline phosphatase as a predictor of hyperbilirubinemia in Egyptian neonates. J Arab Soc Med Res. 2020;15:11-7.

15. Abbasian M, Chaman R, DelvarianZadeh M, Amiri M, Raei M, Norouzi $P$ et al. Investigating the prevalence of calcium deficiency and some of its influencing factors in pregnant women and their neonates [in Persian]. Knowledge Health 2012;7:39-43.

16. Nalbantoglu A, Ovalı F, Nalbantoglu B. Alkaline phosphatase as an early marker of hemolysis in newborns. Pediatr Int. 2011;53:936-938.

17. Elmonem H, El-DinA, Kholeef E, Eldin S. Alkaline phosphatase and haptoglobin as predictor of neonatal jaundice. The Egyptian Journal of Hospital Medicine 2019;74(5):978-987.

18. Satrya R, Hidayat S, Effendi SH, Gurnida DA. Correlation between cord blood bilirubin level and incidence of hyperbilirubinemia in term newborns. Paediatr Indonesia. 2009;49:349-354. 